\title{
ON THE SPECTRAL TYPE OF ONE-PARAMETER GROUPS ON OPERATOR ALGEBRAS ${ }^{1}$
}

\author{
DAVID E. EVANS
}

\begin{abstract}
We show that two unitary representations of $\mathbf{R}$ of very different spectral type can give rise to the same one-parameter group of *-automorphisms of a $C^{*}$-algebra.
\end{abstract}

Arveson [1] has introduced a spectral theory for automorphism groups on $C^{*}$-algebras. However, little seems to be known about the spectral type of such groups.

The fact that two unitary representations of $\mathbf{R}$ of very different spectral properties can give rise to the same one-parameter group of automorphisms of a $C^{*}$-algebra is not surprising as can be seen by the following simple example, due to the referee:

Let $H=l^{2}(\mathbf{N})$ and let $h \in B(H)$ be the compact positive operator "multiplication by $\{1 / n\}_{n=1}^{\infty}$ ". Let $\left\{r_{n}\right\}_{n=1}^{\infty}$ be an enumeration of the rationals in [0, 1] and let $k \in B(H)$ be the positive operator "multiplication by $\left\{r_{n}\right\}_{n=1}^{\infty}$ ".

Then, the operator $(h \otimes 1)+(1 \otimes k)$ on $H \otimes H$ has spectrum equal to [0, 2] while $h \otimes 1$ has spectrum equal to $\{1 / n\}_{n=1}^{\infty} \cup\{0\}$. However, the unitary representations of $\mathbf{R}$ on $H \otimes H$ defined by:

$$
t \rightarrow e^{i t(h \otimes 1)}=e^{i t h} \otimes 1
$$

and

$$
t \rightarrow e^{i t(h \otimes 1+1 \otimes k)}=e^{i t h} \otimes e^{i t k}
$$

give rise to the same one-parameter group of automorphisms of the $C^{*}$-algebra $B(H) \otimes 1$.

The purpose of this paper is to show the surprising result that one can go from singular spectrum to absolutely continuous spectrum.

Let $h$ be a bounded selfadjoint operator on a hilbert space $H$. Then, as in the above example, the operators $h \otimes 1$ and $(h \otimes 1)-(1 \otimes h)($ on $H \otimes H)$ give rise to the same one-parameter group of *-automorphisms of the $C^{*}$-algebra $B(H) \otimes 1$. In fact, if $A$ denotes the $C^{*}$-algebra generated by $h$ and 1 , $h \otimes 1$ and $(h \otimes 1)-(1 \otimes h)$ give rise to the same one-parameter group of

Received by the editors December 17, 1975.

AMS (MOS) subject classifications (1970). Primary 46L05, 46L10, 47B15, 22D10.

Key words and phrases. Operator algebra, representation, spectral type.

${ }^{1}$ This research was carried out at the The Mathematical Institute, Oxford. The author would like to thank E. B. Davies for useful discussions on the problem, and the Science Research Council for their financial support. 
*-automorphisms of the spatial $C^{*}$-tensor product $B(H) \otimes A$, and both unitary groups $e^{i(h \otimes 1) t}$ and $e^{i(h \otimes 1-1 \otimes h) t}$ lie in this algebra. Our main result is thus a consequence of

Proposition 1. There exists a bounded selfadjoint operator $h$ on a hilbert space $H$ such that:

(i) $h$ has singularly continuous spectrum.

(ii) $(h \otimes 1)-(1 \otimes h)$ has absolutely continuous spectrum.

Proof. Let $\mu$ be a positive Radon measure on $\mathbf{R}$ which has compact support and is singularly continuous with respect to Lebesgue measure. Let $H=L^{2}(\mathbf{R}, \mu)$, and $h \in B(H)$ be the bounded, selfadjoint operator "multiplication by the independent variable". To calculate the spectral type of $(h \otimes 1)-(1 \otimes h)$ we first observe that $H \otimes H$ is naturally identified with $L^{2}(\mathbf{R}, \mu \times \mu)$, and that under this identification, $(h \otimes 1)-(1 \otimes h)$ becomes the operator "multiplication by $(x-y)$ ". It is also clear that the spectral resolution $\lambda \rightarrow E_{\lambda}$ of this operator is given by the multiplication operators:

$$
E_{\lambda}(x, y)= \begin{cases}1 & \text { if } x-y \leqslant \lambda \\ 0 & \text { if } x-y>\lambda\end{cases}
$$

Moreover since $\mu \times \mu$ is a finite measure, one sees that $(h \otimes 1)-(1 \otimes h)$ has absolutely continuous spectrum if the spectral measure determined by the constant function 1 on $\mathbf{R}^{2}$ is absolutely continuous with respect to Lebesgue measure. It is seen that this measure is $\mu * \tilde{\mu}$; where if $\nu$ is any Borel measure on $\mathbf{R}$, we denote by $\tilde{\nu}$ the measure $\tilde{\nu}(E)=\overline{\nu(-E)}$. If $\nu$ is absolutely continuous with respect to Lebesgue measure, then so is $\nu * \tilde{\nu}$. The converse is false, as the following proposition shows, and establishes our main result:

Proposition 2. There exists a positive Radon measure $\mu$ on $\mathbf{R}$, with compact support, such that $\mu * \tilde{\mu}$ is absolutely continuous with respect to Lebesgue measure, whilst $\mu$ itself is a singular measure.

Proof. According to Salem [2, p. 265], for each $\alpha \in(0,1)$, and $q>2 / \alpha$, there exists a positive Radon measure $\mu$ on $\mathbf{R}$, supported by a compact subset $K$ of $[0,1]$ having Hausdorff dimension $\alpha$ such that $\hat{\mu} \in L^{q}$. This implies that $K$ is of Lebesgue measure zero, and $\mu$ is a singular measure. Choose $q=4$, and $\frac{1}{2}<\alpha<1$. Then $(\mu * \tilde{\mu})^{\wedge}=|\hat{\mu}|^{2} \in L^{2}$. Hence, $\mu * \tilde{\mu}$ is absolutely continuous with respect to Lebesgue measure, but $\mu$ itself is a singular measure.

We would like to thank the referee for helpful comments with the presentation of the above results, and for the first example.

\section{REFERENCES}

1. W. B. Arveson, On groups of automorphisms of operator algebras, J. Functional Analysis 15 (1974), 217-243. MR 50 \#1016.

2. W. J. Donoghue, Distributions and Fourier transforms, Academic Press, New York, 1969.

School of Theoretical Physics, Dublin Institute for Advanced Studies, Dublin 4, IRELAND

Current address: Institute of Mathematics, University of Oslo, Blindern, Oslo 3, Norway 